\title{
Decay of Capillary Turbulence on the Surface of a Semiquantum Liquid
}

\author{
M. Yu. Brazhnikov*, G. V. Kolmakov*, A. A. Levchenko*, P. V. E. McClintock ${ }^{\dagger}$ and \\ L. P. Mezhov-Deglin* \\ *Institute of Solid State Physics RAS, Chernogolovka, 142432, Russia \\ ${ }^{\dagger}$ Department of Physics, Lancaster University, Lancaster, LA1 4YB, UK
}

\begin{abstract}
We study the free decay of capillary turbulence on the charged surface of liquid hydrogen. Contrary to expectations based on the existing self-similar theory of nonstationary wave turbulent processes in ideal liquid, we find that decay begins from the high frequency end of the spectral range, while most of the energy remains localized at low frequencies. We show that finite damping of the waves changes qualitatively the character of the turbulent decay. Numerical calculations based on this idea agree well with the experimental data.
\end{abstract}

Keywords: Quantum liquids, surface waves, turbulence

PACS: $47.27 .-\mathrm{i}, 47.25 .+\mathrm{i}$

It is known that the nonlinear interaction of capillary waves on the surface of liquid hydrogen is relatively strong, and that liquid hydrogen is a perfect fluid for studies of the turbulence on liquid surface $[1,2]$. The possibility of driving the charged surface of liquid hydrogen directly through the application of electrical forces provides an additional advantage.

We report below the results of our studies of the decay of turbulence in a system of capillary waves on the surface of liquid hydrogen. We have observed that decay of the stationary turbulent spectrum starts in the high frequency domain, with the energy remaining localized in the low frequency range of the turbulent spectrum, i.e. near the driving frequency. At low frequencies the turbulent spectrum remains close to its unperturbed shape for a relatively long period of time after the driving force is switched off. This observation is in a sharp contrast to predictions of the self-similar theory of non-stationary wave turbulent processes (see the review [3]), where evolution of the spectrum is considered in the range of frequencies, at which viscous damping can be neglected. According to this theory, the decay of capillary turbulence should start with the damping of low-frequency surface waves, caused by the cascade transfer of energy to higher frequency scales.

The experimental arrangements were similar to those used in our earlier studies of steady-state turbulence on the charged surface of liquid hydrogen [4]. The measurements were made using an optical cell inside a helium cryostat. Hydrogen was condensed into a cup formed by a bottom capacitor plate and a guard ring $60 \mathrm{~mm}$ in diameter and $6 \mathrm{~mm}$ high. The layer of liquid was $6 \mathrm{~mm}$ thick. The top capacitor plate (a collector $60 \mathrm{~mm}$ in diameter) was located at a distance of $4 \mathrm{~mm}$ above the surface of the liquid. A two-dimensional positively charged layer was created just below the surface of the liquid. The temperature of the liquid was $15.5 \mathrm{~K}$. The waves on the charged surface were excited by a periodic driving voltage applied between the guard ring and the upper electrode. The surface oscillations were detected from the variation of the total power $P(t)$ of a laser beam reflected from the surface, which was measured with a photodetector, sampled with an analogue-to-digital converter, and stored in a computer. Given the size of the light spot, the correlation function $I_{\omega}=\left\langle\left|\eta_{\omega}\right|^{2}\right\rangle$ of the surface elevation $\eta(\mathbf{r}, t)$ in frequency representation is directly proportional to the squared modulus of the Fourier transform of the detected signal, $I_{\omega}=\operatorname{const} P_{\omega}^{2}$ at frequencies above $50 \mathrm{~Hz}$. The instantaneous power spectrum $P_{\omega}^{2}$ of the nonstationary surface oscillations was calculated by application of a shorttime Fourier transform [5] to the measured signal $P(t)$.

To establish the steady turbulent state at the surface of the liquid, an ac driving voltage at a frequency $\omega_{p}$ was applied for $\sim 10 \mathrm{~s}$ (on Fig. 1 the driving frequency is $\omega_{p} / 2 \pi=97 \mathrm{~Hz}$ ). After the driving voltage was switched off, we could observe relaxation oscillations of the surface (Fig. 1 (b) and (c)).

It is clearly evident from Fig. 1 that, during decay of the turbulence, it is the high frequency components of the power spectrum that are damped first. The amplitude of the main peak at the driving frequency remains larger than the amplitudes of peaks at the harmonics all the time, both before and after the driving force is switching off; i.e. the surface of the liquid continues to oscillate mainly at the driving frequency. The wave amplitude in the turbulent distribution decreases homogeneously during the decay, and the power-law dependence of the spectrum persists, even for low frequencies down to $100 \mathrm{~Hz}$. 


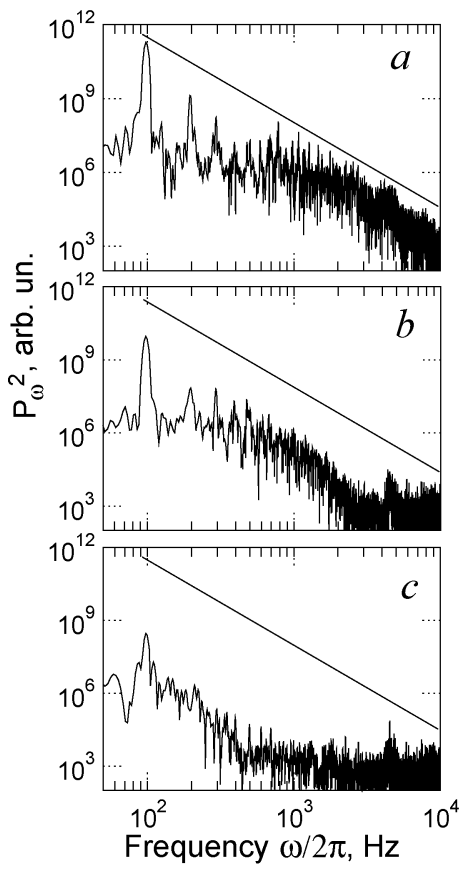

FIGURE 1. Instantaneous spectra of surface oscillations calculated for different moments of time: (a) $t=-0.18 \mathrm{~s}$, i.e. before the removal of the driving force, (b) $t=0.44 \mathrm{~s}$ and (c) $t=1.07 \mathrm{~s}$. Time $t=0$ corresponds to the moment when the driving force was switched off. The straight line corresponds to the power-law dependence $P_{\omega}^{2} \sim \omega^{-7 / 2}$.

To understand better the reason of the apparent discrepancy between our observation and the theoretical predictions we have performed numerical calculations of the capillary turbulence decay, using the kinetic equations for waves (see [3]), in which a viscous damping of waves at all frequencies has been taken into account in addition to the kinetic intergal.

Figures 2 (a) and (b) show the results of our numerical calculations of decay of the turbulent spectra after removal of the narrow-band driving force, for two moments of time corresponding to experimental observations shown in plots (a) and (b) in Fig. 1.

From the results obtained we can claim that, for understanding of nonstationary turbulent processes at the surface of liquid hydrogen, viscous losses in a turbulent system are of central importance at all frequencies above the driving frequency. We have seen, both in experiments and in computations, that finite damping of the waves changes qualitatively the character of the turbulent decay: rather than a propagation of perturbation from low to high frequencies, caused by the cascade transfer of energy (the scenario considered in [3]), there is a relatively fast decay of the high frequency domain of the spectrum. Note that these considerations do not apply to stationary turbulent phenomena, where dissipation can be neglected
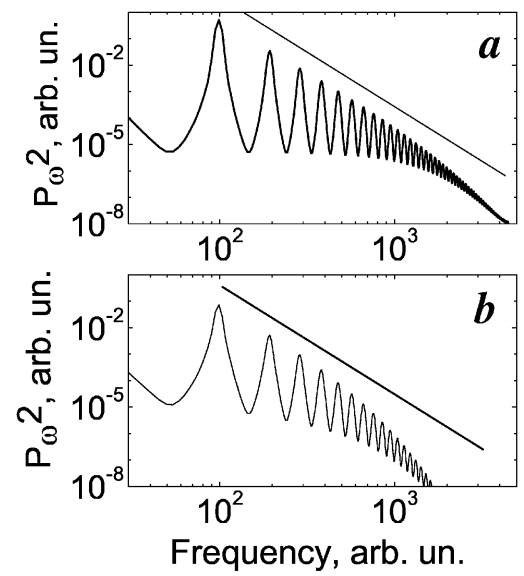

FIGURE 2. Results of our numerical calculations of decay of the turbulent spectra after removal of the narrow-band driving force. Plots (a) and (b) are calculated for moments of time corresponding to the experimental observations shown in plots (a) and (b) on Fig. 1.

completely over an inertial range of frequencies [6].

\section{ACKNOWLEDGMENTS}

The authors are very grateful to V.E. Zakharov, E.A. Kuznetsov, M.T. Levinsen and A.N. Silchenko for many useful discussions, and to V.N. Khlopinskii for assistance in preparation of the experiments. The investigations are supported in part by RFBR (grant 05-02-17849), by the Ministry of Education and Science of RF (program "Quantum macrophysics"), and by the Engineering and Physical Sciences Research Council, UK. M.Yu.B. acknowledges a support from the Science Support Foundation, Russia.

\section{REFERENCES}

1. M.Y. Brazhnikov, G.V. Kolmakov, A.A. Levchenko, and L.P. Mezhov-Deglin, JETP Lett. 73, 398 (2001).

2. G.V. Kolmakov, A.A. Levchenko, M.Yu. Brazhnikov, L.P. Mezhov-Deglin, A.N. Silchenko, and P.V.E. McClintock, Phys. Rev. Lett. 93, 074501 (2004).

3. V. Zakharov, V. L'vov, and G. Falkovich, Kolmogorov Spectra of Turbulence, Vol. 1, Berlin: Springer, 1992.

4. M.Y. Brazhnikov, A.A. Levchenko, and L.P. MezhovDeglin, Instrum. Exp. Tech. 45, 758 (2002).

5. S. Mallat, A Wavelet Tour of Signal Processing, New York: Academic Press, 1997.

6. A. N. Pushkarev and V. E. Zakharov, Phys. Rev. Lett. 76, 3320 (1996). 